\title{
Dependência para alimentar-se e consumo alimentar em idosos hospitalizados
}

\author{
Dependence to feed itself and food consumption in hospitalized elderly \\ Dependencia para alimentarse y consumo alimentario en ancianos hospitalizados
}

' Universidade Estadual de Maringá, Programa de Pós-Graduação em Ciências da Saúde (Mestranda). Maringá-PR, Brasil. "Universidade Estadual de Maringá, Programa de Pós-Graduação em Enfermagem. Núcleo de Estudos, Pesquisa, Assistência e Apoio à Família. Maringá-PR, Brasil.

Submissão: 26-04-2011 Aprovação: 04-01-2013

\section{RESUMO}

O objetivo do estudo foi verificar a associação entre o consumo alimentar e a dependência para alimentar-se em idosos hospitalizados, os quais foram entrevistados nas primeiras $24 \mathrm{hs}$ após internação na clínica médica de um hospital geral. Participaram da pesquisa 75 idosos com idade média de 70,9 anos, sendo $58,7 \%$ do sexo masculino e $17,3 \%$ com dependência para alimentar-se. Não foi evidenciada associação entre dependência para alimentar-se e o consumo de alimentos energéticos e construtores, mas o foi para o consumo dos alimentos reguladores como o alho e cebola $(p=0,00)$ e o de água $(p=0,04)$. Sugere-se a necessidade de orientação nutricional aos cuidadores, visto que a dependência para alimentar-se se associa ao baixo consumo de água entre idosos.

Descritores: Idosos; Consumo Alimentar; Promoção da Saúde.

\section{ABSTRACT}

The objective of the study was to verify the association of food intake and the dependence to be fed in hospitalized elderly individuals who were interviewed in the first 24 hours after admission to a general hospital. Participated in the research 75 individuals average 70.9 years old, being $58.7 \%$ male and $17.3 \%$ showing dependence to be fed. There was no evident association between dependence to be fed and the intake of energetic and muscle building food, but there was an association with regulator food such as garlic and onion $(p=0.00)$ and water $(p=0.04)$. The need of nutritional orientation to the caretakers is suggested, taking into consideration that the feeding dependence is associated to a low intake of water among elderly individuals.

Key words: Elderly; Food Intake; Health Promotion.

\section{RESUMEN}

El objetivo del estudio fue verificar la asociación del consumo alimentario y la dependencia para alimentarse en ancianos hospitalizados, los cuales fueron entrevistados en las primeras $24 \mathrm{hs}$ después de la internación en la clínica médica de un hospital general. Participaron de la investigación 75 ancianos con edad media de 70,9 años, siendo 58,7\% del sexo masculino y $17,3 \%$ con dependencia para alimentarse. No fue evidenciado asociación entre dependencia para alimentarse y el consumo de alimentos energéticos y constructores, pero lo fue para el consumo de los alimentos reguladores como el ajo y cebolla $(p=0,00)$ y el de agua $(p=0,04)$. Se sugiere la necesidad de orientación nutricional a los cuidadores, visto que la dependencia para alimentarse se asocia a bajo consumo de agua entre ancianos.

Palabras clave: Ancianos; Consumo Alimentario; Promoción de la Salud. 


\section{INTRODUÇÃO}

A alimentação é um fator relevante na gênese da má nutrição, desse modo é importante avaliar e monitorar o consumo alimentar em idosos, pois o envelhecimento saudável está associado a uma alimentação adequada à manutenção do estado nutricional.

Dentre os fatores que se associam, direta ou indiretamente, com a quantidade e a qualidade dos alimentos consumidos encontram-se os fisiológicos, sociais, culturais, econômicos e as enfermidades crônicas não transmissíveis ${ }^{(1)}$. Nesse aspecto, a maior ocorrência de enfermidades, com o passar dos anos, representa, para a pessoa idosa, fator adicional, de importância considerável na elaboração de estratégias que visem a orientação nutricional direcionadas ao segmento de idosos ${ }^{(2)}$.

Com o intuito de melhorar a qualidade de vida dos idosos brasileiros o Ministério da Saúde laçou em 2009, um manual dirigido aos profissionais da saúde com orientações sobre alimentação saudável para a pessoa idosa, onde são apresentadas medidas práticas para o preparo e consumo de alimentos, para estimular autonomia do idoso e os dez passos para uma alimentação saudável ${ }^{(3) .}$

O consumo alimentar de idosos é um tema pouco investigado $^{(4-5)}$. A maioria dos estudos focaliza a análise de nutrientes, não levando em consideração o seu consumo. Embora os estudos sobre o consumo de alimentos ${ }^{(1,4,6)}$ sejam escassos, considera-se que essa investigação é fundamental, pois as pessoas consomem alimentos e não nutrientes especificamente, além de que este tipo de investigação possibilita mais facilmente a elaboração de recomendações nutricionais mais efetivas para a prática de saúde pública ${ }^{(7)}$.

A falta de autonomia funcional para preparar e comer alimentos é um fator que pode resultar em desnutrição e merece atenção por parte dos profissionais de saúde e familiares. Uma baixa/deficiente capacidade funcional pode ser um indicador de risco nutricional, visto que este se encontra particularmente associado à ingestão de alimentos ${ }^{(8)}$.

Neste sentido, estudos sobre o consumo alimentar de idosos não devem se restringir somente a análise qualitativa do que é consumido, sendo necessário considerar as particularidades do processo do envelhecimento, como a incapacidade de alimentar-se sozinho. Assim, o objetivo deste estudo foi verificar a associação entre o consumo alimentar e a dependência para alimentar-se em idosos hospitalizados.

\section{METODOLOGIA}

Estudo transversal, de caráter quantitativo desenvolvido em um hospital geral localizado na região noroeste do Paraná. A população em estudo foram os idosos internados na enfermaria da clínica médica e seus respectivos cuidadores, os quais responderam as questões do questionário quando o idoso não conseguia fazê-lo. Foram excluídos os idosos em estado grave com risco de morte. Os dados foram coletados no período de março a junho de 2009, nas primeiras 24hs após a internação do idoso na enfermaria, por meio de entrevista semiestruturada.
Foram utilizados dois instrumentos para a realização da entrevista. $\mathrm{O}$ primeiro foi um questionário estruturado contendo sete questões abordando aspectos sócio-demográficos, uma questão relacionada à capacidade para alimentar-se e três questões referentes ao número de refeições realizadas diariamente, lanches e quantidade de copos de água ingeridos diariamente.

$\mathrm{O}$ segundo instrumento foi um questionário de frequência de consumo alimentar (QFCA), o qual apresenta uma lista de 80 alimentos e a opção referente à frequência de consumo, se diário, semanal, mensal e raramente. Este instrumento foi proposto e validado por Schieri ${ }^{(7)}$, para uso com indivíduos com mais de 12 anos de idade, população adulta e idosa.

A dependência para alimentar-se foi avaliada de acordo com as respostas dadas às perguntas relacionadas com a capacidade para alimentar-se, sendo os idosos classificados em independentes ou dependentes quando necessitavam de algum tipo de ajuda.

Os dados da frequência qualitativa de consumo alimentar foram processados utilizando o "software" Epi Info 3.5.1. Para este estudo foram considerados os 10 alimentos mais frequentemente consumidos diariamente por grupo alimentar (energéticos, construtores, reguladores).

O teste qui-quadrado $\left(\chi^{2}\right)$, ou exato de Fischer, quando aplicável, com nível de significância de 0,05 , foi utilizado para determinar a associação entre o consumo alimentar e a dependência para alimentar-se.

O desenvolvimento do estudo ocorreu em conformidade com o preconizado pela Resolução 196/96 do Conselho Nacional de Saúde e seu projeto foi apreciado pelo Comitê Permanente de Ética em Pesquisa com Seres Humanos da Universidade Estadual de Maringá, (Protocolo nº 090/2009). Todos os participantes assinaram o Termo de Consentimento Livre e Esclarecido em duas vias.

\section{RESULTADOS}

Em relação às características dos 75 idosos participantes do estudo, verifica-se na tabela 1 que mais da metade era do sexo masculino, tinha idade entre 60-69 anos (média de 70,9 anos, $\mathrm{dp}=7,93$ ) e era casado, sendo que uma parcela considerável $(36,0 \%)$ era viúvo. Em relação à escolaridade, 33,3\% eram analfabetos e $58,6 \%$ não chegaram a completar o ensino fundamental. A maioria dos idosos $(82,6 \%)$ apresentava boa cognição e boa condição de comunicação o que possibilitou que os mesmos respondessem aos questionários e por fim, 17,3\% dos idosos apresentavam dependência para alimentar-se.

Quanto aos cuidadores, 84,0\% deles eram do sexo feminino, média de idade de 51,88 anos $(\mathrm{dp}=14,8)$; a maioria tinha baixa escolaridade, sendo que $50,6 \%$ possuía ensino fundamental incompleto, $16,0 \%$ tinham o ensino médio completo, $12,0 \%$ eram analfabetos e 10,6\% tinham o ensino médio incompleto.

A maioria dos acompanhantes tinha algum grau de parentesco com o idoso e, entre os cuidadores do sexo feminino, $27(36,0 \%)$ ocupavam a posição de esposa, 26 (34,6\%) eram filhas, seguidas por netas $(4,0 \%)$ e irmãs $(2,6 \%)$. Já entre os 12 cuidadores do sexo masculino, 5 (6,6\%) eram filhos, dois esposos e um neto. 
Tabela 1 - Distribuição dos idosos hospitalizados segundo características sócio-demográficas, condições de comunicação e dependência para alimentar-se. Sarandi-PR, 2009.

\begin{tabular}{|c|c|c|c|}
\hline \multirow{2}{*}{\multicolumn{2}{|c|}{ Características }} & \multicolumn{2}{|c|}{ Frequência } \\
\hline & & $\mathbf{N}$ &.$(\%)$ \\
\hline \multirow{2}{*}{ Sexo } & Masculino & 44 & 58,7 \\
\hline & Feminino & 31 & 41,3 \\
\hline \multirow{3}{*}{ Idade (anos) } & $60-69$ & 39 & 52,0 \\
\hline & $70-79$ & 23 & 30,7 \\
\hline & 80 ou mais & 13 & 17,3 \\
\hline \multirow{4}{*}{ Estado Civil } & Casado & 39 & 52,0 \\
\hline & Solteiro & 03 & 4,0 \\
\hline & Viúvo & 27 & 36,0 \\
\hline & Divorciado & 06 & 8,0 \\
\hline \multirow{5}{*}{ Escolaridade } & Analfabeto & 25 & 33,3 \\
\hline & Ensino fundamental incompleto & 44 & 58,7 \\
\hline & Ensino fundamental completo & 1 & 1,3 \\
\hline & Ensino médio incompleto & 1 & 1,3 \\
\hline & Ensino médio completo & 4 & 5,3 \\
\hline \multirow{3}{*}{ Comunicação Verbal } & Boa & 62 & 82,7 \\
\hline & Pouca & 12 & 16,0 \\
\hline & Sem & 01 & 1,3 \\
\hline \multirow{2}{*}{$\begin{array}{l}\text { Dependência para } \\
\text { alimentar-se }\end{array}$} & Dependente & 13 & 17,3 \\
\hline & Independente & 62 & 82,7 \\
\hline
\end{tabular}

Tabela 2 - Distribuição dos idosos hospitalizados segundo o consumo dos alimentos energéticos e dependência para alimentar-se, Sarandi-PR, 2009.

\begin{tabular}{|c|c|c|c|c|c|c|}
\hline \multirow[b]{2}{*}{ Alimentos energéticos } & \multirow[b]{2}{*}{$\begin{array}{l}\text { Dependência para } \\
\text { alimentar-se }\end{array}$} & \multicolumn{4}{|c|}{ Consumo alimentar } & \multirow[b]{2}{*}{ p valor } \\
\hline & & $\begin{array}{l}\text { Diário } \\
\text { N (\%) }\end{array}$ & $\begin{array}{l}\text { Semanal } \\
\quad N(\%)\end{array}$ & $\begin{array}{l}\text { Mensal } \\
\text { N (\%) }\end{array}$ & $\begin{array}{l}\text { Raramente } \\
\text { N (\%) }\end{array}$ & \\
\hline Arroz & $\begin{array}{l}\text { Independente } \\
\text { Dependente }\end{array}$ & $\begin{array}{l}58(93,5) \\
11(84,6)\end{array}$ & $\begin{array}{l}4(6,5) \\
1(7,7)\end{array}$ & $1 \stackrel{-}{(7,7)}$ & $\begin{array}{l}- \\
-\end{array}$ & 0,20 \\
\hline Pão & $\begin{array}{l}\text { Independente } \\
\text { Dependente }\end{array}$ & $\begin{array}{l}49(79,0) \\
10(76,9)\end{array}$ & $\begin{array}{l}9(14,5) \\
2(15,4)\end{array}$ & $1(\overline{7,7)}$ & $\begin{array}{c}4(6,5) \\
-\end{array}$ & 0,26 \\
\hline Açúcar & $\begin{array}{l}\text { Independente } \\
\text { Dependente }\end{array}$ & $\begin{array}{l}45(72,6) \\
9(69,2)\end{array}$ & $\begin{array}{c}1(1,6) \\
-\end{array}$ & $\begin{array}{c}1(1,6) \\
-\end{array}$ & $\begin{array}{l}15(24,2) \\
4(30,8)\end{array}$ & 0,81 \\
\hline Margarina & $\begin{array}{l}\text { Independente } \\
\text { Dependente }\end{array}$ & $\begin{array}{c}32(49,2) \\
3(23,1)\end{array}$ & $\begin{array}{c}9(13,9) \\
1(7,7)\end{array}$ & $\begin{array}{l}- \\
-\end{array}$ & $\begin{array}{l}4(36,9) \\
9(69,2)\end{array}$ & 0,09 \\
\hline Macarrão & $\begin{array}{l}\text { Independente } \\
\text { Dependente }\end{array}$ & $\begin{array}{l}7(11,3) \\
4(30,8)\end{array}$ & $\begin{array}{c}46(74,2) \\
9(69,2)\end{array}$ & $\begin{array}{c}6(9,7) \\
-\end{array}$ & $\begin{array}{c}3(4,8) \\
-\end{array}$ & 0,25 \\
\hline Biscoito Doce & $\begin{array}{l}\text { Independente } \\
\text { Dependente }\end{array}$ & $\begin{array}{l}7(11,3) \\
4(30,8)\end{array}$ & $\begin{array}{c}14(22,6) \\
4(30,8)\end{array}$ & $\begin{array}{c}5(8,1) \\
-\end{array}$ & $\begin{array}{c}36(58,0) \\
5(38,4)\end{array}$ & 0,18 \\
\hline Farinha de mandioca & $\begin{array}{l}\text { Independente } \\
\text { Dependente }\end{array}$ & $\begin{array}{c}9(14,5) \\
-\end{array}$ & $\begin{array}{c}19(30,6) \\
3(23,1)\end{array}$ & $\begin{array}{c}8(12,9) \\
-\end{array}$ & $\begin{array}{l}16(42,0) \\
10(76,9)\end{array}$ & 0,12 \\
\hline Balas & $\begin{array}{l}\text { Independente } \\
\text { Dependente }\end{array}$ & $\begin{array}{c}6(9,7) \\
2(15,4)\end{array}$ & $\begin{array}{c}16(25,8) \\
3(23,1)\end{array}$ & $\begin{array}{l}9(14,5) \\
3(23,1)\end{array}$ & $\begin{array}{l}31(50,0) \\
5(38,4)\end{array}$ & 0,67 \\
\hline Chocolate em pó & $\begin{array}{l}\text { Independente } \\
\text { Dependente }\end{array}$ & $\begin{array}{c}5(8,1) \\
2(15,4)\end{array}$ & $\begin{array}{c}7(11,3) \\
1(7,7)\end{array}$ & $\begin{array}{l}3(4,8) \\
1(7,7)\end{array}$ & $\begin{array}{l}47(75,8) \\
9(69,2)\end{array}$ & 0,72 \\
\hline Requeijão & $\begin{array}{l}\text { Independente } \\
\text { Dependente }\end{array}$ & $\begin{array}{l}6(9,7) \\
1(7,7)\end{array}$ & $\begin{array}{c}8(12,9) \\
1(7,7)\end{array}$ & $\begin{array}{c}5(8,1) \\
-\end{array}$ & $\begin{array}{l}43(69,3) \\
11(84,6)\end{array}$ & 0,88 \\
\hline
\end{tabular}

*Teste Exato de Fisher. 
Com relação ao consumo alimentar, entre os 10 alimentos do grupo dos energéticos consumidos diariamente com mais frequência pelos idosos encontra-se o arroz, pão, açúcar, representando $92 \% ; 79 \%$ e $72 \%$ respectivamente.

Não foi evidenciada associação entre o consumo alimentar diário, semanal, mensal e raro com a dependência para alimentar-se em relação aos alimentos energéticos (Tabela 2). Mas, ao analisar o consumo diário dos alimentos energéticos verificou-se maior proporção entre os idosos independentes, exceto pelo consumo diário de macarrão e biscoito doce que apresentaram frequência de 30,8\%, e balas e chocolate em pó correspondendo a $15,4 \%$ do consumo diário dos idosos dependentes.

No consumo diário dos alimentos construtores o feijão apresentou maior frequência entre os idosos correspondendo a $89,3 \%$, seguido do leite $(73,3 \%)$. Apesar de não ter encontrado associação entre o consumo e a dependência para alimentar-se, o leite, frango e o iogurte correspondendo a $84,6 \%, 15,4 \%$ e $7,7 \%$ respectivamente foram os alimentos do consumo diário que apresentaram maior proporção entre os idosos dependentes. Já entre os independentes, os alimentos consumidos com maior frequência foram: feijão $(91,9 \%)$, queijo $(24,2 \%)$, carne vermelha sem osso $(21,0 \%)$, carne com osso $(6,4 \%)$, de porco
$(4,8) \%$, peixes $(4,8 \%)$ e ovos $(3,2 \%)$ (Tabela 3$)$.

Em relação aos alimentos reguladores os mais freqüentes consumidos diariamente foram: o alho $(96,0 \%)$, seguido da cebola $(94,7 \%)$. A cebola e o alho foram os únicos alimentos a apresentarem associação significativa com a dependência para alimentar-se, evidenciado estar mais relacionado ao consumo semanal $(p=0,00)$.

O consumo de alimentos reguladores foram citados com menor frequência pelos idosos dependentes, os quais apresentam consumo diário superior aos idosos independentes apenas em relação a cenoura $(23,2 \%)$ e á maçã $(15,4 \%)$. As frutas banana, laranja e limão são consumidos diariamente pelos idosos independentes, porém apenas a banana é consumida por mais da metade deles (Tabela 4).

No que se refere aos hábitos alimentares dos idosos a maioria $(78,7 \%)$ realizavam três refeições diárias, 22,7\% realizava de dois a três lanches diário e apenas 18,6\% tomavam mais de 8 copos de água por dia. Observou-se que 69,2\% dos idosos dependentes e $80,6 \%$ dos idosos independentes realizavam três refeições diárias e a maioria dos idosos dependentes $(61,5 \%)$ e independentes $(59,7 \%)$ realizavam apenas um lanche diariamente.

Tabela 3 - Distribuição dos idosos hospitalizados segundo o consumo dos alimentos construtores e dependência para alimentar-se, Sarandi-PR, 2009.

\begin{tabular}{|c|c|c|c|c|c|c|}
\hline \multirow[b]{2}{*}{ Alimentos construtores } & \multirow[b]{2}{*}{$\begin{array}{l}\text { Dependência para } \\
\text { alimentar-se }\end{array}$} & \multicolumn{4}{|c|}{ Consumo alimentar } & \multirow[b]{2}{*}{ p valor } \\
\hline & & $\begin{array}{l}\text { Diário } \\
\text { N (\%) }\end{array}$ & $\begin{array}{c}\text { Semanal } \\
\text { N (\%) }\end{array}$ & $\begin{array}{c}\text { Mensal } \\
\text { N (\%) }\end{array}$ & $\begin{array}{l}\text { Raramente } \\
\text { N (\%) }\end{array}$ & \\
\hline \multirow{2}{*}{ Feijão } & Independente & $57(91,9)$ & $3(4,8)$ & - & $2(3,2)$ & \multirow{2}{*}{0,10} \\
\hline & Dependente & $10(76,9)$ & $2(15,4)$ & $1(7,7)$ & - & \\
\hline \multirow{2}{*}{ Leite } & Independente & $44(71,0)$ & $9(14,5)$ & - & $9(14,5)$ & \multirow{2}{*}{0,46} \\
\hline & Dependente & $11(84,6)$ & - & - & $2(15,4)$ & \\
\hline \multirow{2}{*}{ Queijo } & Independente & $15(24,2)$ & $20(32,20$ & $14(22,6)$ & $13(21,0)$ & \multirow{2}{*}{0,46} \\
\hline & Dependente & $1(7,7)$ & $4(30,8)$ & $3(23,1)$ & $5(38,5)$ & \\
\hline \multirow{2}{*}{ Carne sem osso } & Independente & $13(21,0)$ & $45(72,6)$ & - & $4(6,5)$ & \multirow{2}{*}{0,28} \\
\hline & Dependente & $1(7,7)$ & $10(76,9)$ & - & $2(15,4)$ & \\
\hline \multirow{2}{*}{ Frango } & Independente & $6(9,7)$ & $51(82,3)$ & $1(1,6)$ & $4(6,4)$ & \multirow{2}{*}{0,40} \\
\hline & Dependente & $2(15,4)$ & $10(76,9)$ & $1(7,7)$ & - & \\
\hline \multirow{2}{*}{ logurte } & Independente & $4(6,4)$ & $19(30,6)$ & $9(14,5)$ & $30(48,4)$ & \multirow{2}{*}{0,10} \\
\hline & Dependente & $1(7,7)$ & $7(53,8)$ & $3(23,1)$ & $2(15,4)$ & \\
\hline \multirow{2}{*}{ Carne com osso } & Independente & $4(6,4)$ & $8(12,9)$ & $10(16,1)$ & $40(64,5)$ & \multirow{2}{*}{0,29} \\
\hline & Dependente & - & $1(7,7)$ & - & $12(92,3)$ & \\
\hline \multirow{2}{*}{ Carne de porco } & Independente & $3(4,8)$ & $29(46,8)$ & $18(29,0)$ & $12(19,3)$ & \multirow{2}{*}{0,27} \\
\hline & Dependente & - & $4(30,8)$ & $3(23,1)$ & $6(46,1)$ & \\
\hline \multirow{2}{*}{ Peixe fresco } & Independente & $3(4,8)$ & $12(19,3)$ & $28(45,2)$ & $19(19,3)$ & \multirow{2}{*}{1,00} \\
\hline & Dependente & - & $2(15,4)$ & $7(53,8)$ & $4(30,8)$ & \\
\hline \multirow{2}{*}{ Ovos } & Independente & $2(3,2)$ & $30(48,4)$ & $14(22,6)$ & $16(25,8)$ & \multirow{2}{*}{0,89} \\
\hline & Dependente & - & $7(53,8)$ & $2(15,4)$ & $4(30,8)$ & \\
\hline
\end{tabular}

*Teste Exato de Fisher. 
Tabela 4 - Distribuição dos idosos hospitalizados segundo consumo dos alimentos reguladores e dependência para alimentarse, Sarandi-PR, 2009.

\begin{tabular}{|c|c|c|c|c|c|c|}
\hline \multirow[b]{2}{*}{$\begin{array}{l}\text { Alimentos } \\
\text { reguladores }\end{array}$} & \multicolumn{6}{|c|}{ Consumo alimentar } \\
\hline & $\begin{array}{c}\text { Dependência } \\
\text { para alimentar-se }\end{array}$ & $\begin{array}{l}\text { Diário } \\
\text { N (\%) }\end{array}$ & $\begin{array}{l}\text { Semanal } \\
\text { N (\%) }\end{array}$ & $\begin{array}{l}\text { Mensal } \\
\text { N (\%) }\end{array}$ & $\begin{array}{l}\text { Raramente } \\
\quad \text { N (\%) }\end{array}$ & p valor \\
\hline Alho & $\begin{array}{l}\text { Independente } \\
\text { Dependente }\end{array}$ & $\begin{array}{c}60(96,8) \\
6(46,1)\end{array}$ & $6(46,1)$ & $\begin{array}{l}- \\
-\end{array}$ & $\begin{array}{l}2(3,2) \\
1(7,7)\end{array}$ & 0,00 \\
\hline Cebola & $\begin{array}{l}\text { Independente } \\
\text { Dependente }\end{array}$ & $\begin{array}{c}59(94,3) \\
6(46,1)\end{array}$ & $\begin{array}{l}1(1,9) \\
6(46,1)\end{array}$ & $\begin{array}{l}- \\
-\end{array}$ & $\begin{array}{l}2(3,8) \\
1(7,7)\end{array}$ & 0,00 \\
\hline Banana & $\begin{array}{l}\text { Independente } \\
\text { Dependente }\end{array}$ & $\begin{array}{c}36(58,1) \\
6(46,1)\end{array}$ & $\begin{array}{c}24(38,7) \\
7(53,8)\end{array}$ & $\begin{array}{l}- \\
-\end{array}$ & $\begin{array}{c}2(3,2) \\
-\end{array}$ & 0,57 \\
\hline Laranja & $\begin{array}{l}\text { Independente } \\
\text { Dependente }\end{array}$ & $\begin{array}{c}30(48,4) \\
5(38,5)\end{array}$ & $\begin{array}{c}22(35,5) \\
6(46,1)\end{array}$ & $\begin{array}{c}3(4,8) \\
-\end{array}$ & $\begin{array}{l}7(11,3) \\
2(15,4)\end{array}$ & 0,82 \\
\hline Limão & $\begin{array}{l}\text { Independente } \\
\text { Dependente }\end{array}$ & $\begin{array}{c}23(37,1) \\
4(30,8)\end{array}$ & $\begin{array}{c}13(21,0) \\
4(30,8)\end{array}$ & $\begin{array}{c}7(11,3) \\
1(7,7)\end{array}$ & $\begin{array}{l}19(30,6) \\
4(30,8)\end{array}$ & 0,94 \\
\hline Alface & $\begin{array}{l}\text { Independente } \\
\text { Dependente }\end{array}$ & $\begin{array}{c}17(27,4) \\
2(15,4)\end{array}$ & $\begin{array}{c}39(62,9) \\
7(53,8)\end{array}$ & $\begin{array}{c}3(4,8) \\
-\end{array}$ & $\begin{array}{c}3(4,8) \\
4(30,8)\end{array}$ & 0,05 \\
\hline Pimentão & $\begin{array}{l}\text { Independente } \\
\text { Dependente }\end{array}$ & $\begin{array}{c}11(17,7) \\
2(15,4)\end{array}$ & $\begin{array}{c}6(9,7) \\
-\end{array}$ & $\begin{array}{l}11(17,7) \\
2(15,4)\end{array}$ & $\begin{array}{l}34(54,8) \\
9(69,2)\end{array}$ & 0,89 \\
\hline Tomate & $\begin{array}{l}\text { Independente } \\
\text { Dependente }\end{array}$ & $\begin{array}{c}10(16,1) \\
2(15,4)\end{array}$ & $\begin{array}{c}38(61,3) \\
6(46,1)\end{array}$ & $\begin{array}{l}8(12,9) \\
3(23,1)\end{array}$ & $\begin{array}{c}6(9,7) \\
2(15,4)\end{array}$ & 0,60 \\
\hline Cenoura & $\begin{array}{l}\text { Independente } \\
\text { Dependente }\end{array}$ & $\begin{array}{c}6(9,7) \\
3(23,1)\end{array}$ & $\begin{array}{c}35(56,4) \\
9(69,2)\end{array}$ & $\begin{array}{c}10(16,1) \\
-\end{array}$ & $\begin{array}{c}11(17,7) \\
1(7,7)\end{array}$ & 0,20 \\
\hline Maçã & $\begin{array}{l}\text { Independente } \\
\text { Dependente }\end{array}$ & $\begin{array}{c}6(9,7) \\
2(15,4)\end{array}$ & $\begin{array}{l}34(54,8) \\
7(53,8)\end{array}$ & $\begin{array}{l}5(8,1) \\
1(7,7)\end{array}$ & $\begin{array}{c}17(27,4) \\
3(23,1)\end{array}$ & 0,91 \\
\hline
\end{tabular}

Tabela 5 - Distribuição dos idosos hospitalizados segundo os hábitos alimentares e dependência para alimentar-se, Sarandi-PR, 2009.

\begin{tabular}{|c|c|c|c|c|c|}
\hline \multirow{3}{*}{$\begin{array}{c}\text { Hábitos } \\
\text { Alimentares }\end{array}$} & \multicolumn{4}{|c|}{ Dependência para alimentar-se } & \multirow{3}{*}{$\mathbf{p}^{*}$} \\
\hline & \multicolumn{2}{|c|}{ Dependente } & \multicolumn{2}{|c|}{ Independente } & \\
\hline & $\mathbf{N}^{\circ}$ & $\%$ & $\mathrm{~N}^{\circ}$ & $\%$ & \\
\hline \multicolumn{6}{|l|}{ Refeições } \\
\hline 2 & 4 & 30,8 & 12 & 19,4 & \multirow{2}{*}{0,28} \\
\hline 3 & 9 & 69,2 & 50 & 80,6 & \\
\hline \multicolumn{6}{|l|}{ Lanches } \\
\hline Nenhum & 1 & 7,7 & 12 & 19,3 & \multirow{3}{*}{0,57} \\
\hline 1 & 8 & 61,5 & 37 & 59,7 & \\
\hline $2-3$ & 4 & 30,8 & 13 & 21,0 & \\
\hline \multicolumn{6}{|l|}{ Copos água } \\
\hline $2-3$ & 9 & 69,2 & 18 & 29,0 & \multirow{4}{*}{0,04} \\
\hline $4-5$ & 1 & 7,7 & 22 & 35,5 & \\
\hline 6-7 & 1 & 7,7 & 10 & 16,1 & \\
\hline 8 ou mais & 2 & 15,4 & 12 & 19,4 & \\
\hline
\end{tabular}

*Teste Exato de Fisher.
O consumo de água apresentou associação com a dependência para alimentar-se, o que se relacionava, principalmente, ao baixo consumo de água, ou seja, dois a três copos diários $(p=0,04)$. $O$ consumo de dois a três copos de água foi evidenciado em $69,2 \%$ dos idosos dependentes versus $29,0 \%$ dos independentes. Uma minoria dos idosos dependentes $(15,4 \%)$ consumia mais de oito copos de água diário, enquanto $19,4 \%$, dos independentes ingeriam mais de oito copos de água diariamente (Tabela 5).

\section{DISCUSSÃO}

O fato de ter sido identificado que a maioria dos idosos em estudo é do sexo masculino, com baixa escolaridade, um percentual significativo de viúvos e serem cuidados por pessoas também idosas, constitui um indicativo da problemática que envolve a alimentação desse grupo etário. Culturalmente, as mulheres são as responsáveis pelo preparo das refeições, o que pode estar influenciado no consumo e hábitos alimentares dos idosos, especialmente os viúvos.

As mulheres, mais especificamente, as esposas, são responsáveis pela função de cuidar como identificado por vários autores $^{(9-11)}$ e verificado no presente estudo. A existência de cuidadores idosos também corroboram com os resultados de outros estudos que demonstram que idosos estão cuidando de outros idosos $^{(9-10)}$. 
A presença de cuidadores idosos coloca em risco a saúde $\operatorname{destes}^{(10)}$, e demonstra a necessidade de atenção para a própria saúde e qualidade de vida ${ }^{(11)}$. As cuidadoras que vivenciam sobrecarga com atividades domésticas e o cuidado com o idoso podem começar a apresentar estresse, fadiga e maior risco para doenças físicas e emocionais e até depressão ${ }^{(11)}$. Estes fatos levam á reflexão sobre a falta de assistência relacionada à alimentação em nosso meio. Em países desenvolvidos, existem programas, com o propósito de poupar o cuidador de cozinhar todos os dias, nomeada "alimentação sobre rodas", que produz e distribui refeições programadas ${ }^{(9)}$.

Outro aspecto importante a ser considerado é o grau de escolaridade do cuidador, visto que este pode interferir na aprendizagem e, por conseguinte na qualidade da assistência prestada ao idoso ${ }^{(11)}$. Dessa forma, há necessidade de uma orientação nutricional de fácil entendimento, visto a baixa escolaridade e número elevado de cuidadores idosos, já que uma orientação nutricional adequada é eficaz para uma meIhor qualidade de vida.

Considerando que o consumo alimentar de idosos e as particularidades do envelhecimento como a capacidade para alimentar-se constituem assuntos pouco considerados em nosso meio, se faz necessário investigar a existência da associação entre estes fatores, de modo a planejar uma orientação nutricional adequada do ponto de vista da viabilidade econômica e que facilite a rotina e o trabalho dos cuidadores.

No que se refere ao consumo alimentar, pesquisas realizadas em grandes centros urbanos têm apontado uma evolução nos padrões de consumo alimentar, com diminuição no consumo de alimentos básicos e tradicionais como arroz e feijão, aumento de até $400 \%$ no consumo de produtos industrializados, consumo excessivo de açúcar e insuficiente de frutas e hortaliças e aumento no teor da dieta em gorduras em geral e gordura saturada ${ }^{(12)}$. E isto, com certeza tem reflexos no consumo de alimentos de toda a população, inclusive entre os idosos conforme observado.

No grupo dos alimentos energéticos, os consumidos com maior frequência foram o arroz, pão e açúcar, semelhante ao encontrado em outros estudos ${ }^{(1,6,13)}$ que identificaram que o arroz e o pão são consumidos diariamente, com diferenciação por região, onde o arroz foi menos consumido na região de maior poder aquisitivo e o pão na região de menor poder aquisitivo ${ }^{(1)}$.

O consumo diário de macarrão, biscoito doce e chocolate em pó, por idosos dependentes, pode estar relacionado a uma maior facilidade de preparo e menor esforço na mastigação, já que os tipos de preparações melhor aceitas por idosos dependentes são as sopas e papas.

Cabe salientar que em circunstâncias desfavoráveis como isolamento, solidão e comprometimento da capacidade funcional relacionada à alimentação, o consumo de produtos industrializados e de fácil preparo como doces, massas, bolachas e biscoitos são frequentes ${ }^{(14)}$.

No grupo dos alimentos construtores os consumidos com maior frequência diariamente foram o feijão e o leite. Outras leguminosas, no entanto, não são consumidas com frequência pelos idosos, o que diminui a contribuição de proteínas de boa qualidade, o aporte de vitaminas, minerais e fibras ${ }^{(4)}$. Em estudo realizado em São Paulo, verificou-se que 70\% ou mais dos idosos consomem feijão, carnes de boi, aves, leite e substitutos e ovos, porém o feijão e o leite são consumidos diariamente ${ }^{(1)}$. Estudo realizado com mulheres idosas, também encontrou consumo diário de leite entre as idosas, sendo que diariamente, $48 \%$ delas consumiam leite desnatado e $29 \%$ leite integral ${ }^{(6)}$.

Ainda em relação aos alimentos construtores, apesar de não ter sido encontrado associação entre o consumo dos mesmos e a dependência para alimentar-se, é provável que o elevado consumo de frango identificado no presente estudo esteja relacionado ao menor custo, facilidade na utilização em sopas e preparações que facilitam a mastigação e deglutição como, por exemplo, carne desfiada. O mesmo ocorre com o leite que é utilizado na forma de papas, vitaminas e que são alimentos de fácil preparo e aquisição.

Em relação as frutas e hortaliças a Organização Mundial da Saúde recomenda o consumo de cinco ou mais porções diárias, visando prevenir e minimizar a prevalência mundial das doenças crônicas não transmissíveis ${ }^{(15)}$. Entre os alimentos reguladores o alho e a cebola foram os mais citados e isto se deve a utilização dos mesmos como tempero no preparo dos alimentos.

No estudo realizado com idosas em Recife-PE, verificou-se que o consumo de frutas e verduras não foi satisfatório, apesar de mais da metade ( $61 \%$ e $66 \%$ respectivamente) consumirem diariamente esses alimentos ${ }^{(4)}$. Em estudo com 283 idosos de três regiões do município de São Paulo, 85\% deles referiram consumir frutas e verduras, porém ao avaliar o consumo diário observou-se que esta prática é maior em regiões

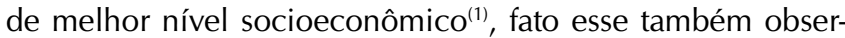
vado em idosos de baixa renda da cidade de São Paulo, onde o consumo inadequado de frutas e verduras esteve associado a menores faixas de renda ${ }^{(5)}$.

No presente estudo, o consumo de frutas e hortaliças foi referido com menor frequência pelos idosos dependentes, o que pode estar associado ao fato de o idoso ser dependente de terceiros tanto para adquirir como para consumir esses alimentos. Além da dificuldade para alimentar-se sozinho, preparar e adquirir esses alimentos, outros fatores associados ao baixo consumo dos mesmos entre os idosos são a escolaridade, inapetência e a presença de doenças crônicas ${ }^{(16)}$.

Apesar das frutas banana, laranja e limão estarem presentes no consumo diário dos idosos independentes, apenas a banana é consumida por mais da metade deles. No estudo realizado com idosos do sexo feminino as frutas: banana, laranja e mamão, embora mais citadas, não apresentaram consumo superior a $40 \%$ entre as idosas ${ }^{(6)}$.

Na terceira idade para uma alimentação saudável, se faz necessário realizar três refeições diárias, intercaladas por lanches, além da ingestão de pelo menos dois litros de água por dia ${ }^{(3)}$. A realização destes hábitos no presente estudo não corrobora aos encontrados em outros estudos ${ }^{(4,6)}$, onde o maior percentual de idosos realizava três refeições diárias, faziam de dois a três lanches diários e ingeriam mais de oito copos de água por dia.

A água constitui elemento importante para o funcionamento do intestino, mantém a boca úmida, o corpo hidratado ${ }^{(3)}$, além de que com o processo de envelhecimento, a quantidade de atividade física, a função renal, as medicações e os 
mecanismos homeostáticos reduzem a sensação de sede e atuam na constipação ${ }^{(17)}$. Outro fator a ser considerado é que o cuidador não deve contabilizar a ingestão de álcool, café e chás na ingestão hídrica do idoso, devido ao efeito diurético dos mesmos, o que poderá contribuir para um maior declínio de água corporal total ${ }^{(18)}$.

Dessa forma, e especialmente considerando a associação estatisticamente significativa entre o consumo de água e á dependência para alimentar-se, é necessário atenção do cuidador para estimular e favorecer o consumo de água entre os idosos dependentes e dos profissionais de saúde para sensibilizar os idosos quanto a importância e os benefícios deste consumo.

\section{CONCLUSÃO}

Os resultados encontrados mostram associação entre consumo alimentar e dependência para alimentar-se em relação ao grupo de alimentos reguladores referente ao alho e cebola e ao baixo consumo de água dos idosos. Apesar de esses alimentos serem utilizados como tempero e, por conseguinte, não apresentarem grande impacto no consumo alimentar e estado nutricional do idoso, constitui preocupação e indica a necessidade de ações mais efetivas referente ao baixo consumo de água nos idosos, oferecendo água ao longo do dia e não substituí-la por bebidas açucaradas e produtos industrializados.

É preciso ainda uma atenção mais cuidadosa em relação a alimentação dos idosos dependentes, pois estes apresentam características peculiares que interferem na ingestão alimentar e quando associada a deficiência na oferta de alimentos pode repercutir negativamente no estado nutricional dos mesmos.

Como os idosos dependentes relataram menor consumo alimentar diário e hábitos alimentares inadequados sugere-se a necessidade de orientação nutricional aos cuidadores, pois a alimentação constitui fator fundamental para a promoção e recuperação da saúde em todas as fases da vida, sendo imprescindível para uma melhor qualidade de vida nos idosos.

\section{REFERÊNCIAS}

1. Najas MS, Andreazza R, Souza ALM, Sachs A, Guedes ACB, Sampaio LR, Ramos LR, Tudisco ES. Padrão alimentar de idosos em diferentes estratos socioeconômicos residentes em localidade urbana da região Sudeste, Brasil. Rev Saúde Pública 1994;28(3):187-91.

2. Marques APO, Arruda IKG, Leal MCC, Santo ACGE. Envelhecimento, obesidade e consumo alimentar de idosos. Rev Bras Geriatr Gerontol 2007;10(2):231-42.

3. Ministério da Saúde. Secretaria de Atenção à Saúde. Departamento de Atenção Básica.Alimentação saudável para a pessoa idosa: um manual para profissionais de saúde / Ministério da saúde, Secretaria de Atenção à Saúde, Departamento de Atenção Básica. - Brasília: Editora do Ministério da Saúde 2009.

4. Fell TCA, Arruda IKG, Ferreira RAR. Aspectos alimentares, nutricionais e de saúde de idosas atendidas no Núcleo de Atenção ao Idoso - NAI, Recife/ 2005. Arq Latino-Americano Nutr 2007;57(4):366-72.

5. Viebig RF, Valero MP, Scazufca M, Menezes PR. Consumo de frutas e hortaliças por idosos de baixa renda na cidade de São Paulo. Rev Saúde Pública 2009;43(5):806-13.

6. Marques APO. Envelhecimento, sobrepeso em mulheres idosas e fatores associados. Recife, 2004. 110 f. Tese (Doutorado) - Universidade Federal de Pernambuco.

7. Sichieri R. Epidemiologia da Obesidade. Rio de Janeiro: EDUERJ; 1998.

8. Schroll M: Aging, food patterns and disability. Forum Nutr 2003;56:256-8.

9. Karsch UM. Idosos dependentes: famílias e cuidadores. Cad Saúde Pública 2003;19(3)861-6.

10. Thober E; Creutzberg M; Viegas K. Nível de dependência de idosos e cuidados no âmbito domiciliar. Rev Bras Enferm 2005;58(4):438-43.
11. Oliveira, SK.; Landgraf-junior, FJ.; Dellaroza, MSG.; Yamada, KN; Trelha, KN.; Cabrera, MAS. Perfil dos cuidadores de idosos atendidos pelo projeto de assistência interdisciplinar a idosos em nível primário - PAINP - Londrina - PR. Ciênc Cuid Saúde 2006;5(2):184-92.

12. Levy-Costa RB., Sichieri R., Pontesc NS., Monteiro CA. Disponibilidade domiciliar de alimentos no Brasil: distribuição e evolução (1974-2003). Rev Saúde Pública 2005;39(4):530-40.

13. Marucci MFN. Aspectos nutricionais e hábitos alimentares de idosos matriculados em ambulatório geriátrico. São Paulo. Tese [Doutorado em Saúde Pública]- Universidade de São Paulo, Faculdade de Saúde Pública; 1992.

14. Moriguti JC, et al. Nutrição no idoso. In: Oliveira JE, Marchine JS. Ciências nutricionais. São Paulo: Savier; 1998. p. 239-51.

15. World Health Organization [homepage na internet] Global Strategy on diet, physical activity and health. Fifty Seventy Word Health Assembly. Geneva; 2004. [acesso em 12 fev 2011]. Disponível em: <http://www.who.int/ dietphysicalactivity/en/>

16. Figueiredo ICR, Jaime PC, Monteiro CA. Fatores associados ao consumo de frutas, legumes e verduras em adultos da cidade de São Paulo. Rev Saúde Pública 2008;42(5):777-85.

17. Arbonés G, Carbajal A, Gonzalvo B, González M, Joyanes M, Marques-Lopes I et al. Nutrición y recomendaciones dietéticas para personas mayores. Grupo de trajo "Salud pública" de La. Sociedad Española de Nutrición (SEN). Nutr Hosp 2003;18:109-37.

18. Russel RM, Rasmussen H, Lichtenstein AH. Modified food guide pyramid for people over seventy years of age. J Nutr 1999;129:751-3. 